\title{
Additional effect of pain neuroscience education to craniocervical manual therapy and exercises for pain intensity and disability in temporomandibular disorders: a study protocol for a randomized controlled trial
}

Aroldo dos Santos Aguiar ${ }^{1}$, Cesar Bataglion², Lilian Ramiro Felício ${ }^{3}$, Beatriz Azevedo ${ }^{1}$ and Thaís Cristina Chaves ${ }^{1,4^{*}}$ (D)

\begin{abstract}
The objective of this study will be to investigate the additional effect of pain neuroscience education program compared to a craniocervical manual therapy and exercises program for pain intensity and disability in patients with temporomandibular disorders (TMD). This study will be a randomized controlled trial comprising a sample of 148 participants. Subjects between 18 and 55 years, both genders, will undergo a screening process to confirm painful TMD by the Research Diagnostic Criteria (RDC/TMD), and then the volunteers will be randomized into two groups (G1: pain neuroscience education + craniocervical manual therapy and exercises vs. G2: craniocervical manual therapy and exercises). The volunteers will be recruited at the dentistry clinic. The intervention will be administered twice a week for 6 weeks by a single therapist lasting $1 \mathrm{~h}$ per session. The primary outcome will be pain intensity and disability and the secondary outcomes will be pain self-efficacy, kinesiophobia, and global perceived effect of improvement. The participants will be assessed immediately after the last session and at oneand three-month follow-ups. All statistical analyses will be conducted following intention-to-treat principles, and the treatment effects will be calculated using linear mixed models. The results of this study may contribute to understand the additional effect of pain neuroscience education intervention on TMD patients submitted to manual therapy and exercise.
\end{abstract}

Trial registration: ClinicalTrials.gov NCT03926767. Registered on April 29, 2019.

Keywords: Temporomandibular joint disorders, Patient education, Exercise therapy, Musculoskeletal manipulations, Chronic pain, Disability evaluation

\footnotetext{
* Correspondence: thaischaves@ufscar.br

'Graduate Program on Rehabilitation and Functional Performance, Ribeirão

Preto Medical School, University of São Paulo, Ribeirão Preto, São Paulo, Brazil

${ }^{4}$ Department of Health Sciences and Graduate Program on Rehabilitation and Functional Performance, Laboratory of Research on Movement and Pain (LabMovePain), Ribeirão Preto Medical School, University of São Paulo, Ribeirão Preto, São Paulo, Brazil

Full list of author information is available at the end of the article
}

(c) The Author(s). 2021 Open Access This article is licensed under a Creative Commons Attribution 4.0 International License, which permits use, sharing, adaptation, distribution and reproduction in any medium or format, as long as you give appropriate credit to the original author(s) and the source, provide a link to the Creative Commons licence, and indicate if changes were made. The images or other third party material in this article are included in the article's Creative Commons licence, unless indicated otherwise in a credit line to the material. If material is not included in the article's Creative Commons licence and your intended use is not permitted by statutory regulation or exceeds the permitted use, you will need to obtain permission directly from the copyright holder. To view a copy of this licence, visit http://creativecommons.org/licenses/by/4.0/. The Creative Commons Public Domain Dedication waiver (http://creativecommons.org/publicdomain/zero/1.0/) applies to the data made available in this article, unless otherwise stated in a credit line to the data. 


\section{Background}

Temporomandibular disorders (TMD) is a collective term that encompasses several clinical diagnoses involving the masticatory muscles, temporomandibular joints (TMJs), and associated structures [1]. The literature recognizes that TMD is not caused by a single factor, it is a complex disorder associated with comorbidities, physical signs and symptoms, changes in behavior, emotional status, social interactions, and changes in the function and structure of the central nervous system [2, 3].

TMD is considered the most frequent cause of chronic orofacial pain of non-dental origin, with a point prevalence of $10-15 \%$ for adults $[4,5]$. In addition, there is a coexistence between TMD and neck pain [6-8], and interventions focused on the craniocervical region as a whole may contribute to decrease pain [9].

Since painful TMD has a multifactorial pathophysiology [2], there is no single approach for treating patients with TMD and the effective management of such disorder has not been established yet [10]. Several studies have demonstrated the effectiveness of interventions like manual therapy associated or not to therapeutic exercise for pain intensity and disability for TMD [9-11]. Greater effect on pain intensity for interventions focused on both orofacial and neck region against placebo/minimal intervention have been reported [9]. Most of the studies included in such systematic reviews showed small sample sizes or adopted a comparison group not submitted to an active treatment.

Current literature shows compelling evidence that the mechanistic biomedical model is not suitable to manage patients with chronic painful TMD [12]. There is a movement towards treatment modalities that encompass the biopsychosocial model that acknowledges and aims to address the biological (physical) and psychosocial factors to treat chronic pain $[13,14]$.

Therapeutic patient education intervention comprises the provision of information to improve the patient's understanding of their problem, speeding up the return to activities and minimizing the dependency of health professionals [15]. Pain neuroscience education (PNE) consists of a set of cognitive interventions whose main objective is to change the patient's conceptualization about pain [16]. A systematic review reported that the combination of PNE with other interventions resulted in more favorable responses for pain intensity, disability and pain catastrophizing in patients with chronic musculoskeletal disorders [17]. Moreover, pain education is the first-line recommendation in clinical practice guidelines for the management of musculoskeletal pain [18, 19].

There is no previous study published in the literature investigating the additional effect of PNE to a protocol of craniocervical manual therapy and exercises for patients with TMD. Then, the objective of our study will be to evaluate the additional effect of PNE to craniocervical manual therapy and exercises on pain intensity and orofacial disability immediately and after 1-month and 3-month follow-ups in patients with TMD. Also, as secondary outcomes, we will investigate the effect of the protocol to pain self-efficacy, kinesiophobia, and global perceived effect of improvement. The hypothesis of this study is that patients submitted to both PNE and manual therapy and exercises will show better outcomes for pain intensity and orofacial-related disability than patients submitted to manual therapy and exercises alone.

\section{Methods}

\section{Trial design}

This study will be a randomized clinical trial with two parallel arms, following the recommendations of the Consolidated Standards of Reporting Trials - CONSORT [20].

\section{Approval and registration}

The study was submitted to and approved by the ethics committee for research involving human subjects of the Clinics Hospital of the Ribeirão Preto, Medical School of the University of São Paulo (HCFMRP Process N ${ }^{\circ} 3449$ / 2018). The study was registered prospectively on Clinical Trials (NCT03926767).

\section{Eligibility criteria and participants}

A sample size of 148 female and male patients with painful TMD will participate in this study. They will be consecutively recruited from the Orofacial Pain Outpatient Clinic from the School of Dentistry of Ribeirão Preto, University of São Paulo. The study is under recruitment and the estimated study completion date is on February 2022. The inclusion criteria for participants were as follows: (i) A diagnosis of painful TMD according to Research Diagnostic Criteria for TMD (RDC/ TMD) [21], (ii) history of orofacial pain at least three months prior to the study [22] and (iii) age ranging between 18 and 55 years, considering the greater prevalence associated with this age period [23].

Illiterate patients, severe depression (medical diagnoses), clinical history of tumors in the craniofacial region, patients in the post dental surgery period or submitted to previous physical therapy in the past year or to any health/pain education strategy, pregnant women, infections, whiplash-associated disorders and with chronic degenerative inflammatory or neurologic disorders were excluded from this study. Patients will be instructed to not use pain relief medications during the intervention period of this trial and if any medication be used, participants will be encouraged to report. All participants will be informed about the procedures of this study, will have 
to report agreement to participate, and will sign the consent form. Participants will be instructed that they will be free to remove consent at any time of the study.

\section{Analysis of the population}

In the event of two consecutive absences from treatment sessions, patients will be contacted by telephone. The analysis will follow the intention-to-treat principles. An intention-to-treat analysis will be performed using the patient's most recent assessment in case of withdrawals or absence of data. Possible adverse effects (injuries) occurring during the intervention period, the individuals will be referred for appropriate treatment.

\section{Procedures, randomization, and allocation}

Once the patient has accepted the invitation to participate and signed the formal consent to participate, one researcher will run the assessment to determine eligibility. After this initial assessment, participants will be randomly assigned following simple computerized randomisation procedures to one of the two treatment groups through the use of cards previously placed in opaque sealed envelopes: G1 - Pain Neuroscience Education + craniocervical manual therapy and exercises or G2 - craniocervical manual therapy and exercises. The allocation sequence will be generated by a researcher not involved in the assessment and interventions (TCC), and another research assistant will assign participants to interventions (Fig. 1). Figure 1 shows the trial procedure, and Table 1 illustrates the trial schedule.

One blinded researcher regarding treatment group allocation will run the assessments pre-treatment, immediately after and at 1-month and 3-month follow-ups. The evaluations of the study will be carried out according to the recommendations of the Initiative on Methods, Measurement and Pain Assessment in Clinical Trials (IMMPACT) for chronic pain [24].

During the assessment for eligibility, the participants will be assessed according to RDC/TMD to confirm the presence of TMD [21]. Afterwards, the participants will answer the Pain Numeric Rating Scale (NPRS) [25], The Craniofacial Pain and Disability Inventory (CF-PDI) [26], Global Perceived Effect (GPE) of improvement [25], Tampa Scale for Kinesiophobia for Temporomandibular Disorders (TSK/TMD )[27], Pain Catastrophizing Scale (PCS) [28], Pain Self-Efficacy Questionnaire (PSEQ) [29], Revised Neurophysiology of Pain Questionnaire (NPQ) [30], and Hospital Anxiety and Depression Scale (HADS) [31]. This protocol followed the Standard Protocol Items: Recommendations for Interventional Trials (SPIRIT) recommendations [32].

\section{Baseline assessment}

Hospital anxiety and depression scale (HADS)

HADS [33] will be employed to identify anxiety disorders and depression. It was translated and validated into Brazilian Portuguese. The HADS is divided into the anxiety subscale (HADS-A) and the depression subscale (HADS-D), both containing seven interspersed items. It is composed of seven items for depression and seven items for anxiety, each item including four response options ranging from 0 to 3 . A cutoff of $\geq 8$ was described with good sensitivity and specificity values $(0.70-0.90)$ for anxiety and depression symptoms [31].

\section{Pain Catastrophizing Scale (PCS)}

The PCS Brazilian Portuguese [28] is a self-administered questionnaire that consists of 13 items for the assessment of catastrophizing thoughts. It is divided into three domains: helplessness, magnification, and rumination. Each item is scored on a 5-point ordinal scale. The BPCS total score ranges from 0 to 52 points, higher values denote greater pain catastrophizing. Acceptable values for validity, internal consistency, and test-retest reliability are described for the Brazilian PCS [28].

\section{Primary outcomes \\ Pain intensity}

The NPRS [25] will be used to assess pain intensity in this trial and consists in a sequence of numbers from 0 to 10 , in which 0 represents "no pain" and 10 represents "worst pain imaginable" [25].

\section{Orofacial and pain disability}

The Craniofacial Pain and Disability Inventory (CF-PDI) [34] is a self-administered questionnaire that measures the outcomes of pain and disability related to craniofacial pain and demonstrated an acceptable structural validity, internal consistency, reproducibility, and construct validity [34]. Also, the Brazilian Portuguese version showed acceptable measurement properties [26]. It consists of 21 items, with a score ranging from 0 to 63 points. Each question is scored on a 4-point ordinal scale, ranging from 0 to 3 . A higher score reflects higher disability levels.

\section{Secondary outcome measures \\ Pain Self-Efficacy Questionnaire -PSEQ}

Study participants will be evaluated on self-efficacy related to chronic pain, which can be defined as an individual's confidence he/she can successfully produce desirable results related to living with chronic pain. The PSEQ has 10 items which are rated on a 7-point ordinal scale (ranging from 0: "not at all confident" to 6: "completely confident"). It was adapted and validated to Brazilian Portuguese [29]. Previous research showed an 


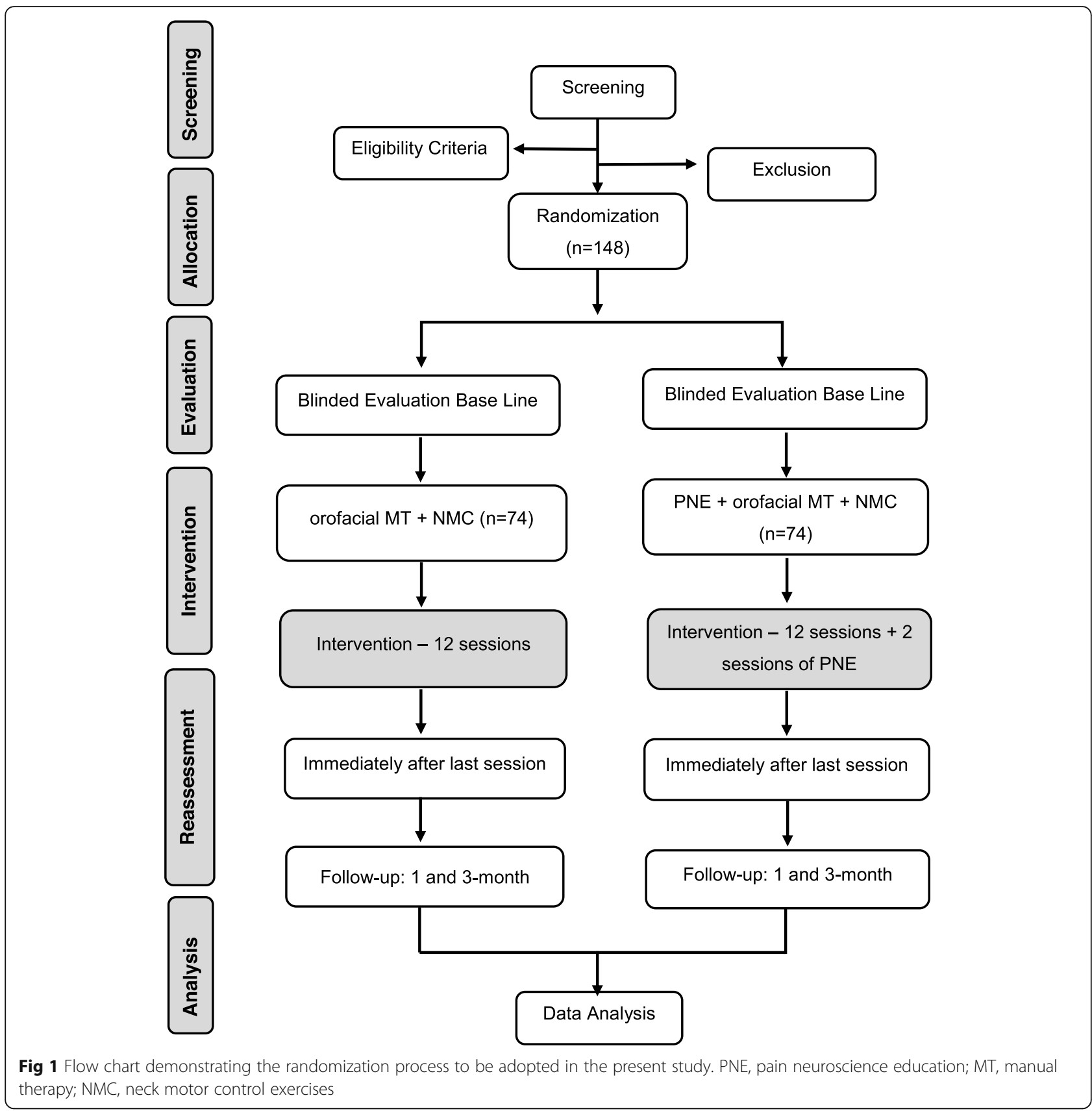

effect on self-efficacy using a PNE intervention based on metaphors compared to an intervention using cognitivebehavioral concepts [35].

\section{Tampa Scale for Kinesiophobia for Temporomandibular Disorders - TSK/TMD}

The TSK/TMD is a self-report questionnaire that assesses the fear of movement [36]. In this study, the TSKTMD with 12 items validated in Brazilian Portuguese was used [27] and showed acceptable psychometric measurements. Each item is scored on a 4-point ordinal scale, ranging from "strongly disagree" $($ score $=1)$ to "strongly agree" (score $=4$ ). Ratings are summed to yield a total score. Higher scores reflect a greater fear of movement (12-48 points).

\section{Participant ratings of Global Perceived Effect - GPE}

The GPE of improvement used for this trial is an 11-point scale that ranges from - 5 ("vastly worse") through 0 ("no change") to +5 ("completely recovered") and participants are asked: "Compared to when this episode first started, how would you describe your orofacial pain these days?". A higher score indicates higher perception of recovery from the condition [25]. 
Table 1 Recommended protocol items: schedule for enrollment, interventions, and assessments

\begin{tabular}{|c|c|c|c|c|c|c|c|c|c|}
\hline & Enrolment & Allocation & Baseline & $\begin{array}{l}\text { Session } \\
1 \text { and } 2 \\
\end{array}$ & $\begin{array}{r}\text { Sess } \\
3 \text { tc }\end{array}$ & ions & $\begin{array}{c}\text { Post } \\
\text { intervention }\end{array}$ & $\begin{array}{c}\text { Follow- } \\
\text { up }\end{array}$ & $\begin{array}{c}\text { Follow- } \\
\text { up }\end{array}$ \\
\hline TIMEPOINT** & $-t_{1}$ & 0 & $t_{1}$ & & \begin{tabular}{l|l|}
3 & 4 \\
\end{tabular} & \begin{tabular}{l|l}
5 & $T$ \\
\end{tabular} & $t_{2}$ & $T_{1}$ & $T_{3}$ \\
\hline ENROLMENT: & & & & & & & & & \\
\hline Eligibility screen & $X$ & & & & & & & & \\
\hline Informed consent & $\mathrm{X}$ & & & & & & & & \\
\hline [Randomization] & & $X$ & & & & & & & \\
\hline Allocation & & $\mathrm{X}$ & & & & & & & \\
\hline INTERVENTIONS: & & & & & & & & & \\
\hline [PNE] & & & & & & & & & \\
\hline $\begin{array}{r}\text { [Orofacial Manual } \\
\text { Therapy] }\end{array}$ & & & & & & & & & \\
\hline $\begin{array}{r}\text { [Orofacial } \\
\text { exercises] }\end{array}$ & & & & & & & & & \\
\hline $\begin{array}{r}\text { [Neck Motor } \\
\text { control exercises] }\end{array}$ & & & & & & & & & \\
\hline ASSESSMENTS: & & & & & & & & & \\
\hline $\begin{array}{r}\text { [Research } \\
\text { Diagnostic Criteria } \\
-R D C / T M D]\end{array}$ & $X$ & & & & & & & & \\
\hline [HADS] & & & $X$ & & & & & & \\
\hline [PCS] & & & $X$ & & & & & & \\
\hline [Pain intensity] & $X$ & & $X$ & & & & $x$ & $X$ & $X$ \\
\hline [CF-PDI] & & & $\mathrm{X}$ & & & & $\mathrm{X}$ & $\mathrm{X}$ & $X$ \\
\hline [PSEQ] & & & $X$ & & & & $X$ & $X$ & $X$ \\
\hline [TSK/TMD] & & & $X$ & & & & $X$ & $X$ & $X$ \\
\hline [NPQ] & & & $X$ & & & & $x$ & & \\
\hline [GPE] & & & & & & & $X$ & $\mathrm{X}$ & $X$ \\
\hline [EARS] & & & & & & & $x$ & & \\
\hline
\end{tabular}

Pain Neuroscience Education (PNE)

Hospital Anxiety and Depression Scale (HADS)

Pain Catastrophizing Scale (PCS)

Cranio Facial Pain and Disability Inventory (CF-PDI)

Pain Self-Efficacy Scale (PSEQ)

Tampa Scale for Kinesiophobia for Temporomandibular Disordes (TSK/TMD)

Neurophysiology of Pain Questionnaire (NPQ)

Global Perceived Effect (GPE)

The Exercise Adherence Rating Scale (EARS)

$P N E=$ pain neuroscience education, HADS = Hospital Anxiety and Depression Scale, PCS = Pain Catastrophizing Scale, CF-PDI = Cranio Facial Pain and Disability Inventory, $P S E Q=$ Pain Self-Efficacy Scale, TSK/TMD = Tampa Scale for Kinesiophobia for Temporomandibular Disordes, NPQ = Neurophysiology of Pain

Questionnaire, GPE = Global Perceived Effect, EARS = The Exercise Adherence Rating Scale 
The revised Neurophysiology of Pain Questionnaire - NPQ The NPQ is a self-administered instrument to assess the knowledge regarding pain neurophysiology. The questionnaire will be administered to quantify the level of knowledge of the participants after the PNE program. Each item has the following response options: true ( 1 point), false ( 0 point), and undecided ( 0 point). The maximum score is 12 points in the revised version. The NPQ showed acceptable internal consistency and good test-retest reliability [37]. There is a cross-culturally version of the NPQ to Brazilian Portuguese [30].

\section{The Exercise Adherence Rating Scale - EARS}

EARS is a patient-reported outcome measure composed of six items that directly assess adherence behavior. In the present study, it will be used to assess the perception of adherence to prescribed home exercises. It was translated and validated into Brazilian Portuguese [38]. The six items should be summed and items with positive phrases are reversely scored, meaning items 1,4 , and 6 . The six items are scored using an ordinal Likert scale of possible answers $(0=$ strongly agree to $4=$ totally disagree), with higher scores indicating greater adherence (0 to 24). Participants will answer the EARS only at the end of the treatment, to assess retrospectively the adherence behavior.

\section{Interventions-general procedure}

On both groups, participants will be submitted to craniofacial manual therapy and an exercise program comprised of Orofacial Exercises and Neck Motor Control Exercises. The participants will be submitted to a program of six weeks of craniofacial manual therapy, orofacial exercises, and neck motor control exercises. The program will be carried out twice a week, one session in the outpatient clinic and another day in the week, the participants will be invited to perform home-based exercises.

Each session will last one hour, always conducted by the same physiotherapist. The first three sessions, participants will be submitted to orofacial manual therapy techniques (myofascial release) and neck motor control exercises (Table 2). And in the remaining sessions, participants will perform half the session orofacial exercises and the other half, neck motor control exercises (Table 2 ). In the first three weeks, only neck motor control exercises (cervical bracing in different positions) will be home-based prescribed. In the last 3 weeks, participants will be instructed to perform orofacial exercises and neck motor control exercises (Table 2).

Table 2 The schedule of the interventions administered in the study. The cervical motor control exercises will be administered according to the progression of the patient in each level

\begin{tabular}{|c|c|c|c|c|}
\hline & & Orofacial manual therapy and exercises & Neck motor control exercises & Pain education \\
\hline \multirow[t]{2}{*}{ Week 1} & Outpatient clinic & Myofascial release (Fig. 2) & $\begin{array}{l}\text { Cervical bracing (Fig. 4) } \\
\text { Neck dynamic isometric exercises (Fig. 5) } \\
\text { Neck functional exercises (Fig. 6) }\end{array}$ & Pain neuroscience education ${ }^{a}$ \\
\hline & Home exercises & & Cervical bracing (Fig. 4) & \\
\hline \multirow[t]{2}{*}{ Week 2} & Outpatient clinic & Myofascial release (Fig. 2) & $\begin{array}{l}\text { Cervical bracing (Fig. 4) } \\
\text { Neck dynamic isometric exercises (Fig. 5) } \\
\text { Neck functional exercises (Fig. 6) }\end{array}$ & Pain neuroscience education ${ }^{a}$ \\
\hline & Home exercises & & Cervical bracing (Fig. 4) & \\
\hline \multirow[t]{2}{*}{ Week 3} & Outpatient clinic & Myofascial release (Fig. 2) & $\begin{array}{l}\text { Cervical bracing (Fig. 4) } \\
\text { Neck dynamic isometric exercises (Fig. 5) } \\
\text { Neck functional exercises (Fig. 6) }\end{array}$ & \\
\hline & Home exercises & & Cervical bracing (Fig. 4) & \\
\hline \multirow[t]{2}{*}{ Week 4} & Outpatient clinic & Mandibular exercises (Fig. 3) & $\begin{array}{l}\text { Cervical bracing (Fig. 4) } \\
\text { Neck dynamic isometric exercises (Fig. 5) } \\
\text { Neck functional exercises (Fig. 6) }\end{array}$ & \\
\hline & Home exercises & Mandibular exercises (Fig. 3) & Cervical bracing (Fig. 4) & \\
\hline \multirow[t]{2}{*}{ Week 5} & Outpatient clinic & Mandibular exercises (Fig. 3) & $\begin{array}{l}\text { Cervical bracing (Fig. 4) } \\
\text { Neck dynamic isometric exercises (Fig. 5) } \\
\text { Neck functional exercises (Fig. 6) }\end{array}$ & \\
\hline & Home exercises & Mandibular exercises (Fig. 3) & Cervical bracing (Fig. 4) & \\
\hline \multirow[t]{2}{*}{ Week 6} & Outpatient clinic & Mandibular exercises (Fig. 3) & $\begin{array}{l}\text { Cervical bracing (Fig. 4) } \\
\text { Neck dynamic isometric exercises (Fig. 5) } \\
\text { Neck functional exercises (Fig. 6) }\end{array}$ & \\
\hline & Home exercises & Mandibular exercises (Fig. 3) & Cervical bracing (Fig. 4) & \\
\hline
\end{tabular}

${ }^{\mathrm{a}}$ Pain education was administered just for one group 


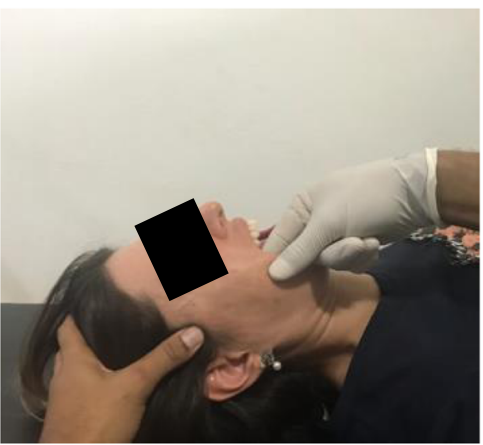

A

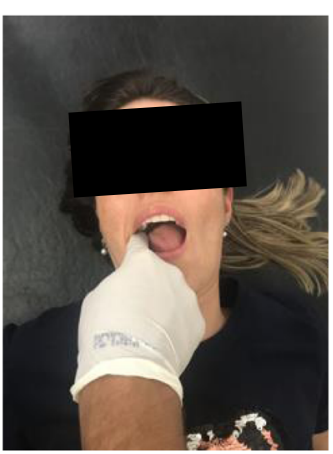

B

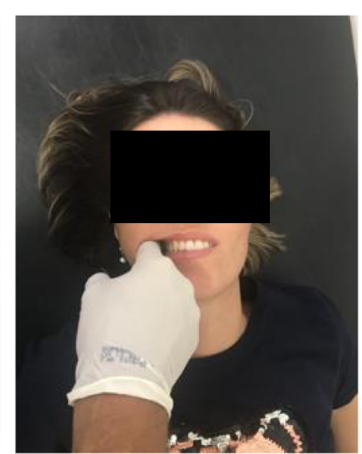

C

Fig. 2 The manual therapy techniques. A Intraoral temporalis myofascial release: The therapist will be positioned by the side in which the technique will be administered, one hand will be on the coronoid process of the mandible with pressure according to the patient's tolerance, while the other hand will be along the temporal muscle. The patient will perform opening movements of the mouth gradually until the maximum opening. B Intraoral medial and lateral pterygoid (origin) technique: The therapist will be positioned contralateral by the side in which the technique will be administered, and with the index finger, the therapist will press the origins of the pterygoid muscles, the pressure will be carefully maintained for $5 \mathrm{~s}$. C Intraoral sphenopalatine ganglion technique: The therapist's index finger will be slowly inserted along the buccal surface of the slightly occluded teeth, the patient will tighten the teeth and, after relaxing, the therapist will apply pressure behind the lingual surface of the masseter and medial pterygoid, this process is repeated until the fingertip approaches the anterior surface of the infratemporal fossa/sphenopalatine fossa, in a comfortable way for the patient

Pain neuroscience education will be administered only to one group in the first and second sessions (half of the session). To balance the clinical attention offered to patients in both groups, the group not submitted to PNE will be invited to clarify possible doubts regarding the home exercises.

\section{The Orofacial Exercises and Orofacial Manual Therapy}

The protocol of Orofacial Exercises and Manual Therapy reported by Kalamir et al. [39] will be adopted in the present study. The manual therapy techniques: intraoral temporalis muscle release, intraoral medial and lateral pterygoid (origin) muscles technique, and intraoral sphenopalatine ganglion technique (Fig. 2). And two mandibular exercises: Mandibular body-condylar crosspressure chewing technique and post-isometric relaxation stretches in jaw laterotrusion and jaw opening (Fig. 3). Each exercise will be executed 10 times per session for $10 \mathrm{~s}$. Participants gave written consent for use of his/her images on pictures 2 to 6 .

\section{Neck motor control exercises}

The neck motor control protocol reported by Celenay et al. [40] will be adopted in our study. The exercise program has three levels: (1) cervical bracing, (2) neck $d y$ namic isometric exercises, and (3) neck functional exercises.

The cervical bracing exercises include four hierarchical levels in neurodevelopment stages (supine, prone, quadrupedal, bipedal) for the cervical spine (Fig. 4). The intermediate level exercises will be comprised of upper and lower extremity range of motion exercises while

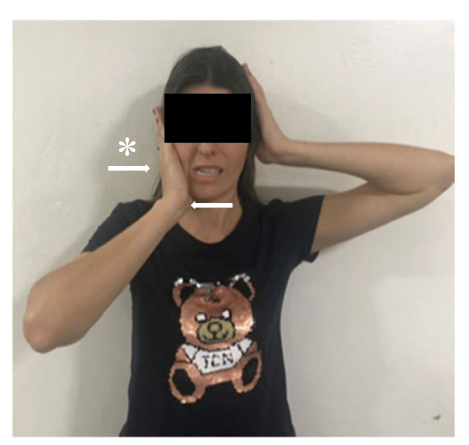

A

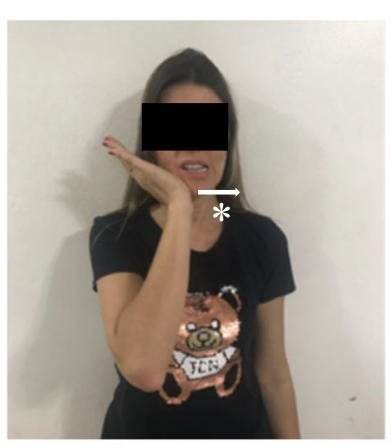

B

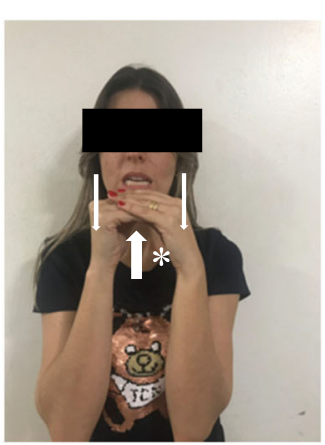

C

Fig. 3 Mandibular exercises. (A) Mandibular body - condylar cross-pressure chewing technique. Post-isometric relaxation stretches - jaw laterotrusion $(\mathbf{B})$ and jaw-opening $(\mathbf{C})$. Arrows indicate the direction of the movements. ${ }^{*}$ Direction of the movement of the hand 


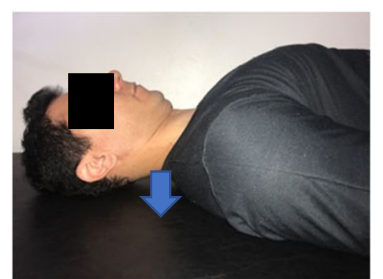

A
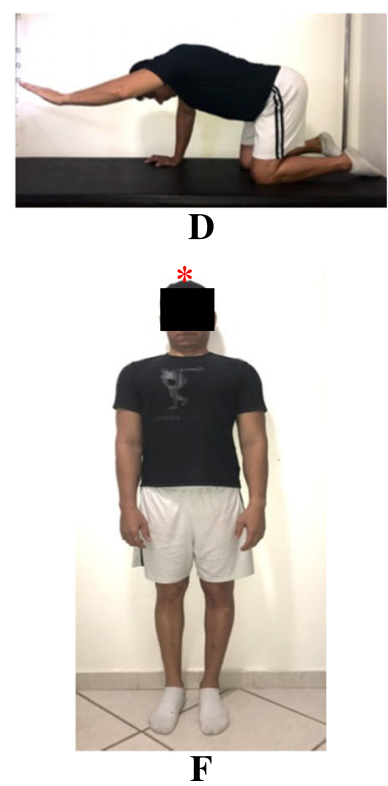

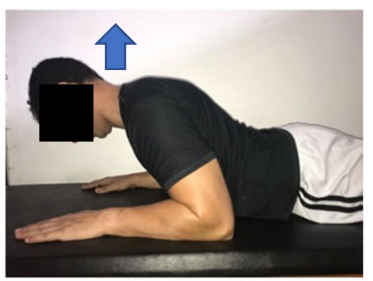

B

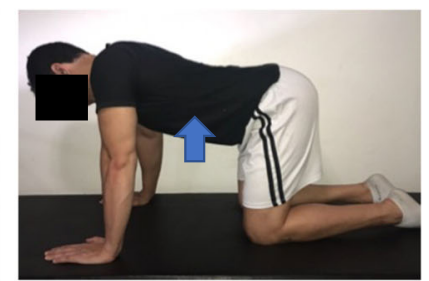

C

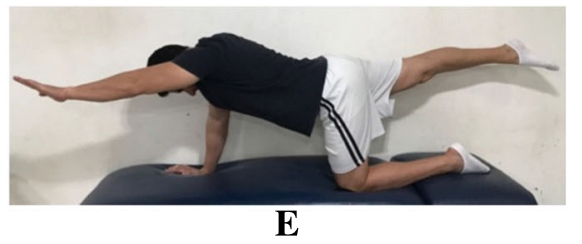

$\mathbf{E}$ extremity range-of-motion movements. F Cervical bracing in the standing position-movement against the wall. Arrows indicate the direction of the movements. * Direction of the movement against the wall

maintaining a stable spine in four-, three-, or two-point kneeling (Fig. 4C-E). The high-level exercise will be the cervical bracing in the upright position (Fig. 4F).

The neck dynamic isometric exercises (five hierarchical levels) will be carried out directly forward, obliquely, toward right and left, and directly backward by maintaining a stable spine with elastic resistive bands (Fig. 5).

Finally, neck functional exercises will include functional training with elastic resistance and exercise balls on unstable surfaces (eight hierarchical levels) (Fig. 6A-H). The criteria to progress in each level (cervical bracing, neck dynamic isometric exercises, and neck functional exercises) will be holding the contraction, for $10 \mathrm{~s}, 10$ times. The progression of the exercises adopted will follow the sequence described on Figs. 4, 5, and 6.

Participants will be oriented to contract deep neck flexor muscles only during the exercises, not throughout the day as described in the protocol of the original paper [40].

\section{Pain neuroscience education (PNE)}

All participants in the G1 will initially receive two additional sessions in which a workshop on PNE will be administered and discussed. A power-point presentation with metaphors and animated videos will be employed. The PNE program will be held in 2 sessions of $40 \mathrm{~min}$ each. The intervention program will be divided into 17 thematic topics according to explain pain [41]:

1. Contextualization on the importance of the program: how pain causes suffering and its alarming increase in the world

2. Initial concepts on neurosciences and pain

3. How context can influence pain perception-incredible stories on pain

4. Human beings as a multisensory complex-sensory information is reaching the brain all the time

5. Pain output may be deflagrated by memory, not only nociception

6. Nociception and the concept of pain as a response of protection/the nociceptors

7. The incorrect concepts on pain (pain system, pain receptors, pain area in the brain)

8. Concepts on pain neurophysiology-synapses, spinal horn, primary and secondary order neurons

9. Types of sensitization/descending nociception modulation system 


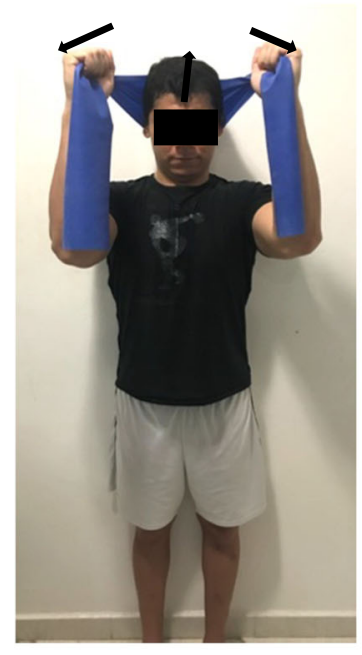

A

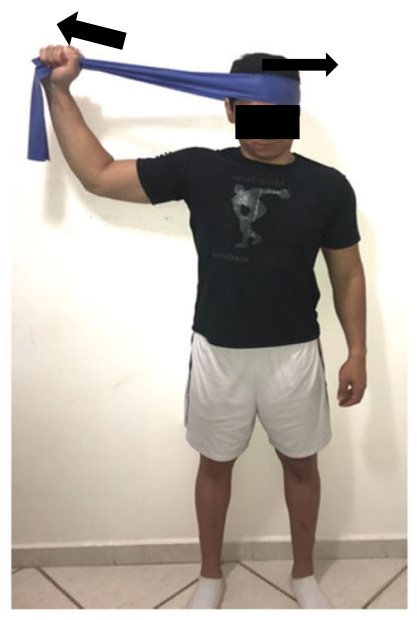

B

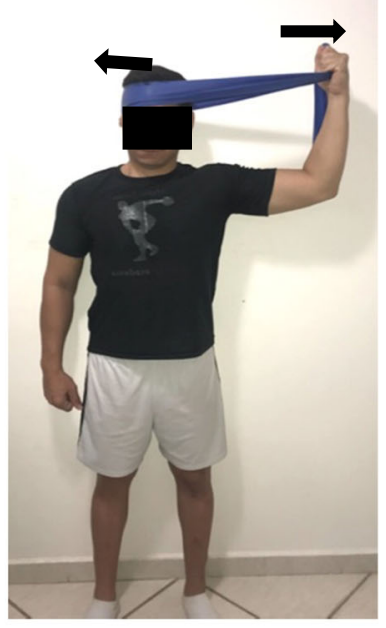

C

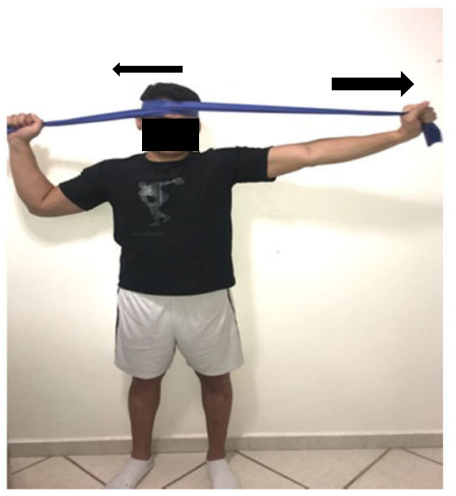

D

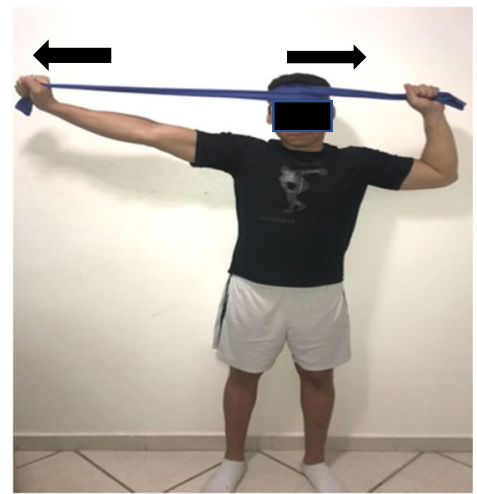

E

Fig. 5 Neck motor control exercises program—neck dynamic isometric exercises were performed directly forward (A), obliquely, toward right (C) and left (B), and directly backward by maintaining a stable spine with elastic resistive bands (D, E). Arrows indicate the direction of the movements

10. The danger message and the brain processing

11. The sensitized brain and its relationship to chronic pain

12. The contribution of other systems to pain experience and vice-versa: endocrine, parasympathetic/sympathetic, immune, and motor

13. How bone, muscles, and nerves send sensory information all the time

14. Fear-avoidance model revisited

15. Encouragement to change

16. How to develop positive attitudes

17. Concepts of graded exposure and graded activity (at least two questions). We will reconceptualize the beliefs of the questions with higher scores on TSK/TMD. A schedule will be developed together with the patient to favor patient exposition to activities they used to avoid.

\section{Sample size calculation}

The study was designed to detect a between-group difference of 2 points in pain intensity (30\%) measured by the NPRS, with an estimated standard deviation of 3 points. A previous study [42] recommended a minimum sample size of $n=61$ per group.

For disability, a between-group difference of 5.08 points for disability measured by the CF-PDI, considering the Smallest Detectable Change of CF-PDI Brazilian version [26], with an estimated standard deviation of 10 points resulted in a sample size of 64 participants per group. The other specifications were power of $80 \%$, an alpha of 5\%, $f$ test of 0.33 (pain intensity) and 0.25 (disability). Therefore, a total of 128 participants was obtained, however considering a follow-up loss up to $15 \%$, we will recruit 148 participants. G*Power was used to 


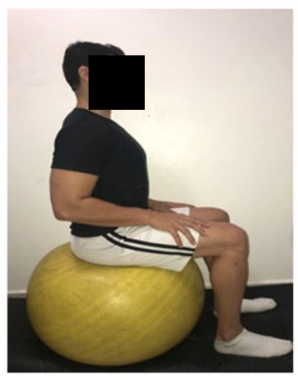

A

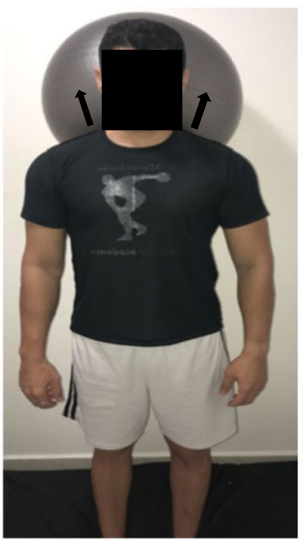

D

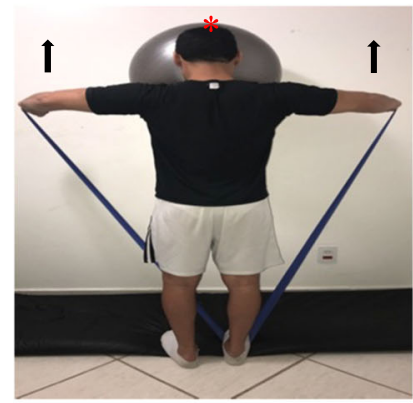

G

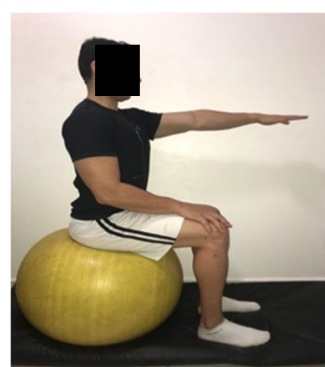

B

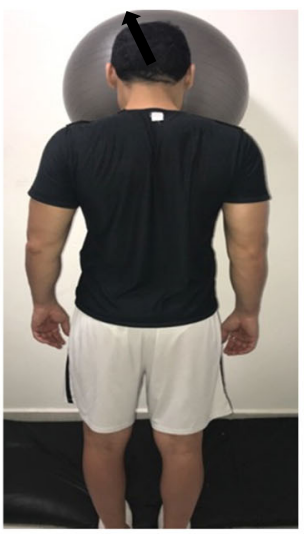

$\mathbf{E}$

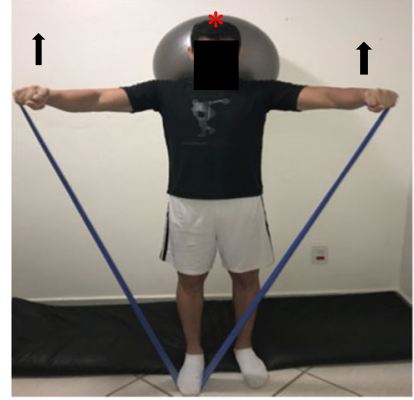

$\mathbf{H}$

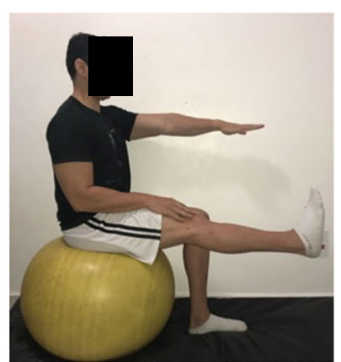

C

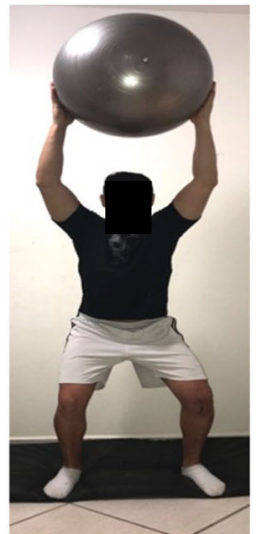

$\mathbf{F}$

Fig. 6 Neck motor control exercises program_functional training on unstable surface was performed in combination with cervical bracing over the ball $(\mathbf{A}-\mathbf{C})$, with ball in standing position $(\mathbf{D}-\mathbf{F})$ and with ball and elastic resistance $(\mathbf{G}, \mathbf{H})$. Arrows indicate the direction of the movements. * Direction of the movement against the wall

run the sample size calculation (GPower 3.0.10, da University of Kiel, Germany).

\section{Data analysis}

The mean effects of the interventions and the group differences for all outcomes (primary and secondary) will be calculated using linear mixed models, incorporating terms for the treatment groups, time (post-intervention and follow-up), and interaction terms (treatment subgroups and time) as well as, psychosocial variables, knowledge about pain neurophysiology (score on the NPQ), sex, ethnicity, and age as covariates. Secondary analysis will be conducted using regression models to determine whether baseline scores of psychosocial variables (HADS, PCS, TSK/TMD) will moderate the effect of treatments. All data will be double entered prior to the analysis. The analysis will follow the intention-to-treat principles. Additionally, we will adopt analysis per protocol excluding patients that did not complete the 6 weeks of treatment. The statistician will receive coded data and will be blinded to the participants' allocation groups. The data collected will be stored coded to protect patient confidentiality. In order do to handle missing data, we will replace the value by the last observation carried forward. For all analysis, we will use the IBM SPSS software 
package, version 22 (IBM Corp, Armonk, NY) and the significance level will be established at 0.05 .

\section{Discussion}

This study will be the first randomized controlled trial in which the additional effect of PNE to Orofacial Manual Therapy/Orofacial exercises/Neck Motor Control exercises will be investigated in patients with chronic TMD pain for the outcomes of pain intensity and orofacial-related disability. Also, to the best of our knowledge, there is no previous study in the literature that investigated the additional effect of PNE program in patients with TMD. This study will help to better understand if PNE will add a significant effect (immediate and at follow-up) to a manual therapy/movement therapy protocol in patients with chronic pain TMD.

In physiotherapy clinical practice it is a common approach to deliver manual therapy + exercises to manage TMD patients. The previous systematic review with meta-analysis showed an effect of manual therapy for pain intensity and disability compared to other interventions $[10,11,43]$ or when orofacial manual therapy is associated with neck exercises [9]. However, it is noteworthy a generalized problem of small sample size for the studies conducted in the field. Moreover, no study was conducted to assess the effect of adding a psychosocial intervention to manual therapy and therapeutic exercise for TMD patients.

In order to put in to practice a biopsychosocial approach in the chronic pain field, it is mandatory the conciliation of education strategies focused on psychosocial factors and interventions related to movement therapy. There are several systematic reviews showing promising effects of PNE for chronic pain patients [17, 44]. A meta-analysis showed that the addition of PNE to other interventions showed a significant effect on pain intensity, disability, and catastrophizing [17].

In this way, this study will help to bring several contributions to the literature studies available in the literature: (i) the initiative to incorporate PNE to TMD treatments commonly delivered; (ii) the assessment of the effect of PNE on psychosocial outcomes such as kinesiophobia and self-efficacy in TMD patients; and (iii) the use of both orofacial manual therapy and orofacial and neck exercises to treat patients with chronic TMD.

\section{Trial status}

Protocol number: NCT03926767. Registered April 29, 2019. Recruiting: Study start

date: April 24, 2019. Study completion date: February 2022.

\section{Abbreviations}

TMD: Temporomandibular disorders; PNE: Pain neuroscience education; HADS: Hospital Anxiety and Depression Scale; NPRS: Pain Numeric Rating
Scale; PCS: Pain Catastrophizing Scale; CF-PDI: Craniofacial Pain and Disability Inventory; PSEQ: Pain Self-Efficacy Scale; TSKTTMD: Tampa Scale for Kinesiophobia for Temporomandibular Disorders; NPQ: Neurophysiology of Pain Questionnaire; GPE: Global Perceived Effect; EARS: The Exercise Adherence Rating Scale

\section{Acknowledgements \\ Not applicable.}

\section{Authors' contributions}

TCC and ASA analyzed, interpreted the data, and written the paper. ASA and BA participated in data collection. TCC, CB, LRF, and ASA reviewed the paper. All authors read and approved the final manuscript.

\section{Funding}

None.

\section{Availability of data and materials}

The datasets used and/or analyzed during the current study are available from the corresponding author on reasonable request.

\section{Declarations}

Ethics approval and consent to participate

This study was approved by the Ethics Committee Board of the Hospital das Clínicas da Faculdade de Medicina de Ribeirão Preto - University of São Paulo (process number: 3449/2019). Informed consent will be obtained by researchers from all study participants.

\section{Consent for publication}

Participants gave written consent for use of his/her images on pictures 2 to 6.

\section{Competing interests}

The authors declare that they have no competing interests.

\section{Author details}

${ }^{1}$ Graduate Program on Rehabilitation and Functional Performance, Ribeirão Preto Medical School, University of São Paulo, Ribeirão Preto, São Paulo, Brazil. ${ }^{2}$ Department of Restorative Dentistry at Ribeirão Preto Dental School (FORP), University of São Paulo-USP, Ribeirão Preto, São Paulo, Brazil. ${ }^{3}$ Graduate Program of Physical Therapy, Laboratory of Evaluation in Biomechanics and Neurosciences (LABiN), Federal University of Uberlândia, Uberlândia, Minas Gerais, Brazil. ${ }^{4}$ Department of Health Sciences and Graduate Program on Rehabilitation and Functional Performance, Laboratory of Research on Movement and Pain (LabMovePain), Ribeirão Preto Medical School, University of São Paulo, Ribeirão Preto, São Paulo, Brazil.

Received: 17 December 2020 Accepted: 11 August 2021

Published online: 06 September 2021

\section{References}

1. Okeson JP, de Leeuw R. Differential diagnosis of temporomandibular disorders and other orofacial pain disorders. Dent Clin N Am. 2011;45(1): 105-20.

2. Lin CS. Brain signature of chronic orofacial pain: a systematic review and meta-analysis on neuroimaging research of trigeminal neuropathic pain and temporomandibular joint disorders. PLoS One. 2014;9(4):e94300.

3. Slade GD, Ohrbach R, Greenspan JD, et al. Painful Temporomandibular disorders: Decade of discovery from OPPERA studies. J Dent Res. 2016;95: 1084-92.

4. Macfarlane TV, Glenny AM, Worthington HV. Systematic review of population-based epidemiological studies of oro-facial pain. J Dent. 2001; 29(7):451-67.

5. List T, Jensen RH. Temporomandibular disorders: Old ideas and new concepts. Cephalalgia. 2017;37(7):692-704.

6. Armijo-Olivo S, Magee DJ, Parfitt M, Mojor P, Thie NM. The association between the cervical spine, the stomatognathic system and craniofacial pain: a critical review. J Orofac Pain. 2006;20(4):271-87. 
7. Wiesinger B, Malker H, Englund E, Wanman A. Does a dose-response relation exist between spinal pain and temporomandibular disorders? BMC Musculosketal Disorders. 2009;10:28.

8. Testa M, Geri T, Gizzi L, Petzke F, Falla D. alterations in masticatory muscle activation in people with persistent neck pain despite the absence of orofacial pain or temporomandibular disorders. J Oral Facial Pain Headache. 2015;29(4):340-8

9. La Touche R, Martínez García S, Serrano García B, Proy Acosta A, Adraos Juárez D, Fernández Pérez JJ, Angulo-Díaz-Parreño S, Cuenca-Martínez F, Paris-Alemany A, Suso-Martí L. Effect of Manual Therapy and Therapeutic Exercise Applied to the Cervical Region on Pain and Pressure Pain Sensitivity in Patients with Temporomandibular Disorders: A Systematic Review and Meta-analysis. Pain Med. 2020;21(10):2373-84.

10. Martins WR, Blasczyk JC, Aparecida Furlan de Oliveira M, et al. Efficacy of musculoskeletal manual approach in the treatment of temporomandibular joint disorder: A systematic review with meta-analysis. Man Ther. 2016;21: 10-7.

11. Dickerson SM, Weaver JM, Boyson AN, Thacker JA, Junak AA, Ritzline PD, et al. The effectiveness of exercise therapy for temporomandibular dysfunction: a systematic review and meta-analysis. Clin Rehabil. 2017;31(8): 1039-48.

12. Gil-Martínez A, Grande-Alonso M, López-de-Uralde-Villanueva I, López-López A, Fernández-Carnero J, La Touche R. Chronic Temporomandibular Disorders: disability, pain intensity and fear of movement. J Headache Pain. 2016:17(1):103

13. Booth J, Moseley GL, Schiltenwolf M, Cashin A, Davies M, Hübscher M Exercise for chronic musculoskeletal pain: A biopsychosocial approach. Musculoskeletal Care. 2017;15(4):413-21.

14. Albano MG, d'Ivernois JF, de Andrade V, Levy G. Patient education in dental medicine: A review of the literature. Eur J Dent Educ. 2019;23(2):110-8.

15. Clarke CL, Ryan CG, Martin DJ. Pain neurophysiology education for the management of individuals with chronic low back pain: systematic review and meta-analysis. Man Ther. 2011;16(5):544-9.

16. Moseley GL, Butler D. Fifteen Years of Explaining Pain: The Past, Present, and Future. J Pain. 2015;16(9):807-13.

17. Watson JA, Ryan CG, Cooper L, et al. Pain Neuroscience Education for Adults With Chronic Musculoskeletal Pain: A Mixed-Methods Systematic Review and Meta-Analysis. J Pain. 2019;20(10):1140 e1-1140.e22.

18. Babatunde OO, Jordan JL, Van der Windt DA, Hill JC, Foster NE, Protheroe J. Effective treatment options for musculoskeletal pain in primary care: A systematic overview of current evidence. PLoS One. 2017;12(6):e0178621.

19. Lin I, Wiles $L$, Waller $R$, et al. What does best practice care for musculoskeletal pain look like? Eleven consistent recommendations from high-quality clinical practice guidelines: systematic review. Br J Sports Med. 2020;54(2):79-86

20. Moher D, Hopewell S, Schulz KF, Montori V, Gøtzsche PC, Devereaux PJ, et al. Consolidated Standards of Reporting Trials Group CONSORT 2010 Explanation and Elaboration: Updated guidelines for reporting parallel group randomised trials. J Clin Epidemiol. 2010;63(8):e1-37.

21. Dworkin SF, LeResche L. Research Diagnostic Criteria for temporomandibular disorders: review, criteria, examinations and specifications, critique. J Craniomandib Disord. 1992;6:301-55.

22. Treede RD, Rief W, Barke A, Aziz Q, Bennett Ml, Benoliel R, et al. A classification of chronic pain for ICD-11. Pain. 2015;156:1003-7.

23. Lövgren A, Häggman-Henrikson B, Visscher CM, Lobbezoo F, Marklund S, Wänman A. Temporomandibular pain and jaw dysfunction at different ages covering the lifespan--A population-based study. Eur J Pain. 2016;20(4):53240.

24. Dworkin RH, Turk DC, Farrar JT, Haythornthwaite JA, Jensen MP, Katz NP, et al. Core outcome measures for chronic pain clinical trials: IMMPACT recommendations. Pain. 2005;113:9-19.

25. Costa LO, Maher CG, Latimer J, Ferreira PH, Ferreira ML, Pozzi GC, et al. Clinimetric testing of three self-report outcome measures for low back pain patients in Brazil: which one is the best? Spine (Phila Pa 1976). 2008:33(22): 2459-63.

26. Greghi SM, Dos Santos AA, Bataglion C, Ferracini GN, La Touche R, Chaves TC. Brazilian Portuguese Version of the Craniofacial Pain and Disability Inventory: Cross-Cultural Reliability, Internal Consistency, and Construct and Structural Validity. J Oral Facial Pain Headache. 2018;32(4):389.

27. Aguiar AS, Visscher CM, Bevilaqua-Grossi D, Bataglion C, Chaves TC. Crosscultural adaptation, reliability and construct validity of the Tampa Scale for kinesiophobia for Temporomandibular Disorders (TSK/MD-BR) into Brazilian Portuguese. J Oral Rehabil. 2017:44(7):500-10.

28. Sehn F, Chachamovich E, Vidor LP, et al. Cross-cultural adaptation and validation of the Brazilian Portuguese version of the pain catastrophizing scale. Pain Med. 2012;13:1425-35

29. Sardá J, Nicholas MK, Pimenta CA, Asgharl A. Pain-related self-efficacy beliefs in a Brazilian chronic pain patient sample: a psychometric analysis. Stress Health. 2007;23(3):185-90.

30. Nogueira LAC, Chaves AO, Nathalia O, Almeida RS, Reis FJJ, Andrade FG, et al. Cross-cultural adaptation of the Revised Neurophysiology of Pain Questionnaire into Brazilian Portuquese language. Jornal Brasileiro de Psiquiatria. 2018;67(4):273-7.

31. Bjelland I, Dahl AA, Haug TT, Neckelmann D. The validity of the Hospital Anxiety and Depression Scale. An updated literature review. J Psychosom Res. 2002;52:69-77.

32. Chan AW, Tetzlaff JM, Altman DG, et al. SPIRIT 2013 statement: defining standard protocol items for clinical trials. Ann Intern Med. 2013;158(3):200-7.

33. Botega NJ, Bio MR, Zomignani MA, Garcia Júnior C, Pereira WA. Transtornos do humor em enfermaria de clínica média e validação de escala de medida (HAD) de ansiedade e depressão. Rev Saude Publica. 1995;29:355-63.

34. La Touche R, Pardo-Montero J, Gil-Martinez A, et al. Craniofacial pain and disability inventory (CF-PDI): development and psychometric validation of a new questionnaire. Pain Physician. 2014;17:95

35. Gallagher L, McAuley J, Moseley GL. A randomized controlled trial of using a book of metaphors to reconceptualize pain and decrease catastrophizing in people with chronic pain. Clin J Pain. 2013:29:20-5.

36. Visscher CM, Ohrbach R, van Wijk AJ, Wilkosz M, Naeije M. The Tampa Scale for Kinesiophobia for Temporomandibular Disorders (TSK-TMD). Pain. 2010; 150(3):492-500

37. Catley MJ, O'Connell NE, Moseley GL. How good is the neurophysiology of pain questionnaire? A Rasch analysis of psychometric properties. J Pain. 2013;14(8):818-27.

38. Lira MR, de Oliveira AS, França RA, Pereira AC, Godfrey EL, Chaves TC. The Brazilian Portuguese version of the Exercise Adherence Rating Scale (EARSBr) showed acceptable reliability, validity and responsiveness in chronic low back pain. BMC Musculoskelet Disord. 2020;21(1):294.

39. Kalamir A, Bonello R, Graham P, Vitiello AL, Pollard H. Intraoral myofascial therapy for chronic myogenous temporomandibular disorder: a randomized controlled trial. J Manipulative Physiol Ther. 2012;35(1):26-37.

40. Celenay ST, Akbayrak T, Kaya DO. A Comparison of the effects of stabilization exercises plus manual therapy to those of stabilization exercises alone in patients with nonspecific mechanical neck pain: a randomized clinical trial. J Orthop Sports Phys Ther. 2016;46(2):44-55.

41. Butler D, Moseley GL. Explain Pain, 2nd ed. Adelaide: Australia, Noigroup Publications; 2013. p. 135.

42. Dao TT, Lavigne GJ, Feine JS, Tanguay R, Lund JP. Power and sample size calculations for clinical trials of myofascial pain of jaw muscles. J Dent Res. 1991:70(2):118-22.

43. Calixtre LB, Moreira RF, Franchini GH, Alburquerque-Sendín F, Oliveira AB. Manual therapy for the management of pain and limited range of motion in subjects with signs and symptoms of temporomandibular disorder: a systematic review of randomised controlled trials. J Oral Rehabil. 2015; 42(11):847-61.

44. Wood L, Hendrick PA. A systematic review and meta-analysis of pain neuroscience education for chronic low back pain: Short-and long-term outcomes of pain and disability. Eur J Pain. 2019;23(2):234-49.

\section{Publisher's Note}

Springer Nature remains neutral with regard to jurisdictional claims in published maps and institutional affiliations. 